\title{
Quantifying Forearm Soft Tissue Motion from Massless Skin Markers following Forward Fall Hand Impacts
}

\author{
Danielle L. Gyemi ${ }^{1}$, Don Clarke', Paula M. van Wyk ${ }^{1}$, William J. Altenhof ${ }^{2}$, David M. Andrews ${ }^{1 *}$ \\ ${ }^{1}$ Department of Kinesiology, University of Windsor, 401 Sunset Avenue, Windsor, ON, Canada, N9B 3P4 \\ ${ }^{2}$ Department of Mechanical, Automotive and Materials Engineering, University of Windsor, 401 Sunset Avenue, Windsor, ON Canada, \\ N9B $3 P 4$
}

Corresponding Author: David M. Andrews, E-mail: dandrews@uwindsor.ca

This research was funded by the Natural Sciences and Engineering Research Council of Canada (NSERC).

\section{ARTICLE INFO}

\section{Article history}

Received: May 05, 2018

Accepted: July 03, 2018

Published: July 31, 2018

Volume: 6 Issue: 3

Conflicts of interest: None

Funding: NSERC

\begin{abstract}
Background: Investigating soft tissue motion related to impact events is important for understanding how the body mitigates potentially injurious forces through shock attenuation. Objectives: The aims of this study were to: 1) quantify displacement and velocity of the forearm soft tissues following forward fall impacts; and 2) compare two massless skin marker designs (single layer, uniform (SLU) design; stacked, non-uniform (SNU) design) in terms of how well they could be tracked over varying skin pigmentations using automated motion capture software. Methods: Two participant groups (skin pigmentation: light - 9F, 8M; dark - 9F, 6M) underwent simulated forward fall hand impacts for each marker design using a torso-release apparatus. Marker positions associated with planar motion of forearm soft tissues during impact were automatically tracked (ProAnalyst ${ }^{\mathbb{R}}$ ) in the proximal-distal and anterior-posterior axes from high speed recordings $(5000 \mathrm{f} / \mathrm{s})$. Mean peak displacements and velocities for eight forearm regions were then calculated (LabVIEW ${ }^{\circledR}$ ). Results: Overall, soft tissue displacement and velocity increased from distal to proximal forearm regions. The greatest displacement $(1.47 \mathrm{~cm})$ and velocity $(112.8 \mathrm{~cm} / \mathrm{s})$ occurred distally toward the wrist. Soft tissue impact responses between sexes did not differ, on average $(p>0.05)$. The SLU and SNU markers produced different kinematic values $(p<0.05)$; however, the magnitudes of, and consequently meaningfulness of these statistical differences for automatically tracking soft tissue motion, were negligible (displacement: $\leq 0.05 \mathrm{~cm}$; velocity: $\leq$ $2.5 \mathrm{~cm} / \mathrm{s}$ ). Conclusions: Forearm soft tissue motion was successfully quantified for forward fall hand impacts; both marker designs were deemed functionally equivalent.
\end{abstract}

Key words: Upper Extremity, Forearm, Accidental Falls, Biomechanical Phenomena, Pattern Recognition, Automated

\section{INTRODUCTION}

Impacts to the hands and wrists resulting from forward falls, whether accidental in nature or due to recreational sporting activities, are problematic in both young and older adult populations because of the high incidence of upper extremity injuries (e.g., sprains, dislocations, fractures) associated with them (Nevitt \& Cummings, 1993; Idzikowski, Janes \& Abbott, 2000; Palvanen et al., 2000; Mirhadi, Ashwood \& Karagkevrekis, 2015). Research concerning the injury mechanisms of a forward fall onto the hands of outstretched arms has largely focused on the in vitro impact response of the distal radius and its ability to dissipate high levels of mechanical energy (Myers et al., 1991; Muller, Webber \& Bouxsein, 2003; Burkhart, Andrews \& Dunning, 2012). However, the movement of soft tissue masses (muscle, fat, skin) relative to bone also plays a protective role in mitigating the injurious effects of impact through shock attenua- tion (Cole, Nigg, van den Bogert \& Gerritsen, 1996; Pain \& Challis, 2002; Gittoes, Brewin \& Kerwin, 2006; Pain \& Challis, 2006). Compared to impact events involving the lower extremity (e.g., running, drop landings) (Cole et al., 1996; Gittoes et al., 2006; Pain \& Challis, 2006), very limited information exists regarding soft tissue shock attenuation for upper extremity impacts to date (Pain \& Challis, 2002).

Various motion tracking techniques have been employed to quantify soft tissue motion (predominantly in the lower extremity) for human movement analysis, including 3D optoelectronic systems (Fuller, Liu, Murphy \& Mann, 1997; Gao \& Zheng, 2008; Akbarshahi et al., 2010; Wolf \& Senesh, 2011), magnetic resonance imaging (Sangeux, Marin, Charleux, Dürselen \& Ho Ba Tho, 2006), as well as radiological methods such as X-ray and video fluoroscopy (Sati, Guise, Larouche \& Drouin, 1996; Südhoff, Van Driessche, Laporte, de Guise \& Skall, 2007; Wrbaškić \& Dowling, 
2007; Akbarshahi et al., 2010; Kuo et al., 2011). A noted major limitation across each of these methods is the need to affix external devices (e.g., accelerometers, active or passive surface markers) to the body segment in order to track soft tissue motion; an action shown to alter natural physiological soft tissue movement following impact (Leardini, Chiari \& Croce, 2005; Stefanczyk, Brydges, Burkhart, Altenhof \& Andrews 2013). Thus, utilizing motion tracking techniques that do not require external devices is key to prevent interference with the soft tissue impact response.

One such technique was presented in studies by Stefanczyk et al. (2013) and Brydges, Burkhart, Altenhof and Andrews (2015), in which position and velocity data of leg soft tissue motion following pendulum and drop landing heel impacts was quantified using massless skin markers and motion capture software with automatic feature tracking capabilities (ProAnalyst ${ }^{\mathbb{R}}$; Xcitex, Cambridge, MA, USA). The marker design ( $2 \times 2 \mathrm{~cm}$ grid of $0.5 \mathrm{~cm}$ diameter black dots $)$ required no mechanical interaction with the leg, and was applied using flexible plastic stencils and a permanent black marker pen. Overall, Brydges et al. (2015) reported good to acceptable reliability for this technique. Nonetheless, modifications to marker shape and contrast might help to further improve its capacity to automatically track soft tissue motion and reduce measurement error (Haddadi \& Belhabib, 2008; Crammond, Boyd \& Dulieu-Barton, 2013), especially across different skin pigmentations.

Therefore, the aims of this study were to: 1) quantify planar displacement and velocity of the forearm soft tissues following a forward fall impact, and assess if there were differences between sexes or as a function of forearm region measured; and 2) determine if a stacked, non-uniform (SNU) marker design (non-uniform, $\sim 0.5 \mathrm{~cm}$ diameter black dots overlaid on top of a grid of contrasting $\sim 1 \mathrm{~cm}$ diameter white dots; $2 \mathrm{~cm}$ inter-marker distance) produced significantly different kinematic results and/or improved automated marker tracking across different skin pigmentations compared to the single layer, uniform (SLU) marker design (grid of uniform, $0.5 \mathrm{~cm}$ diameter black dots; $2 \mathrm{~cm}$ inter-marker distance) previously established by Stefanczyk et al. (2013) and Brydges et al. (2015).

\section{METHODS}

\section{Participants and Study Design}

The repeated-measures design of the present experimental study involved thirty-two (18 female, 14 male) healthy, young adult participants (mean [SD] age, height, and body mass of 22.3 [2.8] years, 1.73 [0.09] $\mathrm{m}$, and 71.2 [14.0] kg, respectively), who were right hand dominant and free of upper extremity pain or injury over the previous year (as indicated on a general health questionnaire). Prior to testing, all aspects of the study were communicated and informed consent was obtained from each participant. All methods and experimental procedures were approved by the participating university.

A modified Fitzpatrick Skin Type Questionnaire (i.e., a numerical classification system for human skin color found- ed on genetic disposition and the reaction of different skin types to ultraviolet light (Fitzpatrick, 1988)) was used to categorize participants into either a light (Type I-III: 9 female, 8 male) or dark (Type IV-VI: 9 female, 6 male) skin pigmentation group. Effort was made to match participants between groups according to height $(\mathrm{m})$, body mass $(\mathrm{kg})$, age (17-30 years), and sex.

\section{Impact Apparatus}

Bilateral hand impacts consistent with a forward fall onto the hands of outstretched arms were applied using a torso-release apparatus (Figure 1). Wearing a fitted safety harness connected to a tether, participants stood on an elevated platform $(72 \times 9 \times 4 \mathrm{~cm})$, which helped prevent ankle plantarflexion during the fall (Kim \& Ashton-Miller, 2003). Each participant started the impact trials in a slight forward lean (approximately $10^{\circ}$ ) with their shoulders flexed to $90^{\circ}$ and arms outstretched in front of their body. A manually-controlled quick release device securely affixed to a heavy, steel frame acted as an attachment point for the tether to support the participant's body weight. Across all impact trials, a distance of approximately $30 \mathrm{~cm}$ was maintained between the thenar regions of participants' palms and two force plates, which were mounted rigidly to a vertical steel structure attached to the steel framing of the laboratory wall. The force plates were positioned side-by-side at a $20^{\circ}$ angle from vertical to simulate the positions of the wrist $\left(\sim 45^{\circ}\right.$ extension) and forearm ( $\sim 75^{\circ}$ with respect to the ground), characteristic of a forward fall (Myers et al., 1991; Greenwald, Janes, Swanson \& McDonald, 1998; Burkhart et al., 2012; Burkhart, Quenneville, Dunning \& Andrews, 2014). Targets were also outlined on the force plates to help standardize the impact postures for each participant. From the start position, participants were manually quick-released by the investigator after a random time delay between 0 and 5 seconds. Participants were instructed to maintain vertical alignment of their head, trunk, and lower extremities for the

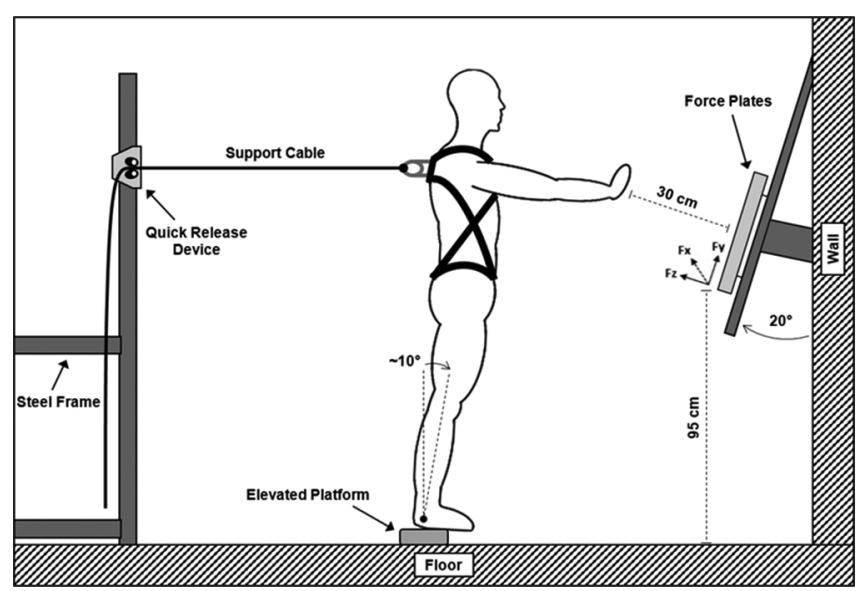

Figure 1. An illustration of the experimental test set-up from a lateral view showing the torso release apparatus and location of the force plates. The diagram depicts the positioning of the participant prior to initiating the forward fall simulation. Note that there were two force plates mounted side-by-side, one for each hand to impact. 
entirety of the impact trial by imitating the fall of a broomstick (Kim \& Ashton-Miller, 2003; Hwang, Kim, Kaufman, Cooney \& An, 2006), and arrest the fall with their arms in full elbow extension (i.e., stiff-arm landing) (Robinovitch \& Chiu, 1998; DeGoede \& AstonMiller, 2002a).

\section{Instrumentation}

Force data for each hand during the impact trials were collected using two force plates (AMTI-OR6-6-1000, A-Tech Instruments Ltd., Scarborough, ON, Canada; $1000 \mathrm{~Hz}$ natural frequency) (Figure 2). A high speed camera (FASTCAM SA4, Photron USA, Inc., San Diego, CA, USA; 5000 frames/s, 1024 x 800 pixels $^{2}$ resolution, shutter speed $0.2 \mathrm{~ms}$ ) was used to record planar soft tissue motion of the lateral aspect of the pronated right forearm. Two industrial-grade spot lights (T1 Fresnel, ARRI, Munich, Germany; 1000W, $120 \mathrm{~V})$ were positioned above and below the forearm to ensure ample lighting for video capture. A non-contact laser displacement transducer (AR700-50, Acuity $^{\circledR}$, Schmitt Measurement Systems, Inc., Portland, OR, USA; sampling rate $9 \mathrm{kHz}$ ), configured along the same plane as the force plates, was used to trigger and synchronize the collection of force and video data during the impact trials, such that the participants' hands would cross the laser beam approximately $1 \mathrm{~cm}$ before contacting the surface of the force plates.

\section{Procedures}

The SLU marker design was tested first (Figure 3a and $b$ ). With the right forearm in pronation, a flexible plastic stencil was wrapped around the posterior and lateral surface of each participant's forearm and the markers were applied using a black permanent marker pen. A designated row along the midline of the posterior forearm (first marker just lateral to the styloid process of the ulna) was used to maintain consistent marker placement between participants; the number of marker columns depended on the forearm length from the wrist to the elbow joint. The SNU marker design was tested second (Figure $3 \mathrm{c}$ and d), and was manually drawn over top of the SLU marker design on the right forearm using white and black water-based paint marker pens. The same investigator applied both marker designs for all participants. If necessary, any hair interfering with the marker application was shaved.

Each participant then underwent a minimum of three impact trials per marker design; a total of six trials were used for subsequent analyses (SLU: impact trials 1-3; SNU impact trials 4-6). Three-dimensional impact forces $\left(F_{x}, F_{y}\right.$, and $F_{z}$ : see coordinate system in Figure 1 for orientation) were recorded at each hand. However, based on the bilateral symmetry of the impact forces and small magnitudes of $F_{x}$ and $F_{y}$, only mean peak $F_{z}$ at the right hand was reported. To ensure consistent impacts between marker designs, the variability of the impact forces across all six impact trials was limited to a range of $10 \%$ to $15 \%$ of each participant's body weight. Trials that fell outside of this range were mostly attributable to improper body postures at impact (e.g., elbow flexion), and thus, were repeated. The to- tal number of trials executed did not exceed more than six per marker design.

\section{Video Analysis}

Videos of forearm soft tissue motion were imported into ProAnalyst ${ }^{\circledR}$ motion tracking software and were subjected to the same calibration process to set the scale and coordinate system for automated tracking. To convert pixels to centimetres

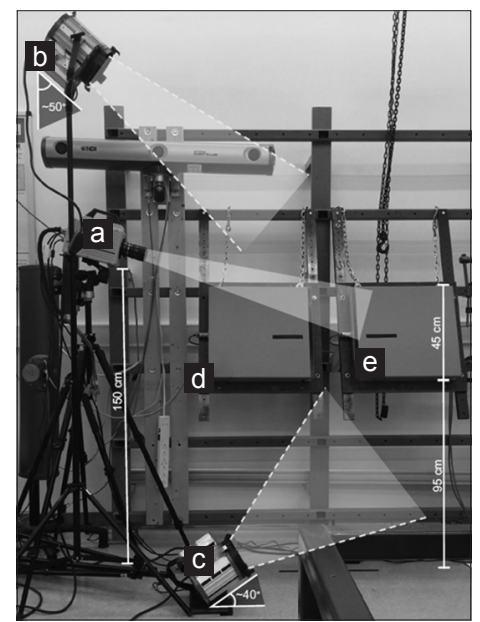

Figure 2. Schematic diagram of the experimental test set-up: a) high-speed camera; b) primary flood light; c) secondary flood light; d) left hand force plate; e) right hand force plate

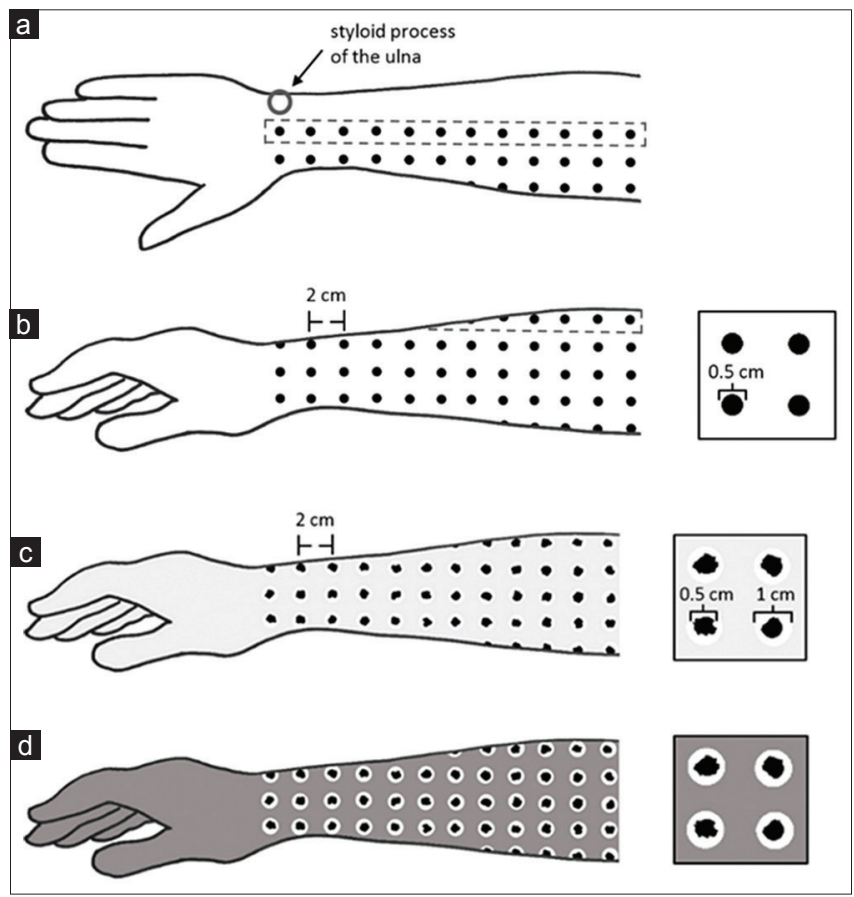

Figure 3. Schematic diagram of the SLU marker design $(2 \times 2 \mathrm{~cm}$ square grid of $0.5 \mathrm{~cm}$ diameter circular black dots) on the forearm from (a) posterior and (b) lateral views; schematic diagram of the SNU marker design $(2 \times 2 \mathrm{~cm}$ square grid of $\sim 1 \mathrm{~cm}$ diameter circular white dots with $\sim 0.5 \mathrm{~cm}$ diameter random black dots overlaid on top) on the forearm demonstrating the contrast for (c) light and (d) dark skin pigmentations. 
a calibration unit of $6 \mathrm{~cm}$ was applied between four adjacent markers (in the same row) that were directly distal to the elbow (Stefanczyk et al., 2013; Brydges et al., 2015); the planar (2D) axes of the coordinate system were aligned in two directions ( $x$-axis: parallel with the long axis of the radius and ulna, running in the proximal-distal direction; $y$-axis: perpendicular to the long axis of the radius and ulna, running in the anterior-posterior direction of the forearm) (Figure 4). A single image filter (Convolve: Sharpening ( 3 x 3 Center)) was applied using the default settings to slightly enhance the overall sharpness of the raw video footage for better contrast and marker edge detection.

The grid of markers on the forearm was segmented into four zones, similar to Brydges et al. (2015) for the leg, wherein two columns of markers (A and B) were selected at $0 \%, 25 \%, 50 \%$, and $75 \%$ of the distance from the styloid process of the ulna to the elbow joint (Figure 4). Each marker location was manually selected by the investigator, ensuring that the rectangular boundary of the defined search region was as close to the marker edge as possible. Following appropriate marker selection, automated motion tracking was performed in ProAnalyst ${ }^{\circledR}$ (search region multiplier $-125 \%$; threshold tolerance -0.75 ) for each impact trial, in which the centre point of all defined regions (i.e., selected markers) was tracked, and the $x$ and $y$ position coordinates were subsequently outputted. The search parameters were held the same for all markers that were automatically tracked. Analysis of the markers began just prior to the right palm impacting the force plate and continued until forearm soft tissue motion following impact had ceased; a duration of approximately 100 to $230 \mathrm{~ms}$ (500 to 1150 frames) across all participants.

\section{Data Analysis}

Marker position coordinates from ProAnalyst ${ }^{\mathbb{}}$ were imported into a custom LabVIEW program $\left(\mathrm{LabVIEW}^{\circledR}\right.$ 2016, National Instruments, Austin, TX, USA) where they were converted to displacement data and filtered using a dual-pass, fourth-order Butterworth low-pass digital filter (Stefanczyk et al., 2013; Brydges et al., 2015) with a cut-off frequency of $60 \mathrm{~Hz}$, determined by residual analysis (Winter, 2005). Velocity data were calculated using a $2^{\text {nd }}$ order central differentiation method (Equation 1). Overall, 10 impact response parameters were assessed in relation to the planar movement of forearm soft tissue following forward fall hand impacts: peak displacement $(\mathrm{cm})$ and velocity $(\mathrm{cm} / \mathrm{s})$ in the proximal-distal and anterior-posterior directions, as well as two additional variables of proximal and posterior rebound distance $(\mathrm{cm})$ (i.e., the distance the marker rebounded from peak displacement in the distal and anterior directions, respectively). To account for potential differences in these impact responses due to soft tissue distribution, each of the four zones $(0 \%, 25 \%$, $50 \%, 75 \%$ ) were further split into anterior and posterior regions by visually dividing the forearm in half, creating a total of eight separate regions along the forearm to be analyzed and ensuring that a relatively equal number of markers were allocated to both sides (Figure 5). For each participant, a single marker was randomly selected within each of these regions for soft tissue kinematic analyses; the same marker was used for both the SLU and SNU marker designs.

$$
\frac{\mathrm{dx}}{\mathrm{dt}}=\frac{\mathrm{x}_{i+1}-\mathrm{x}_{i-1}}{2 \Delta \mathrm{t}}
$$

for $i=0,1,2, \ldots, n-1$

where $n$ is the number of samples.

In order to isolate forearm soft tissue motion caused by impact consistently across all trials and participants, a specific onset point was selected at which to start the analysis of the filtered kinematic data. The onset point was specified as the moment the right palm fully contacted the force plate and the forearm ceased the "free-fall" phase of the fall simulation. The onset point corresponded to a "knee point" in the proximal-distal velocity curve where the velocity in the distal direction began to rapidly decrease from a relatively constant value. For each participant, the onset point was based on the kinematic data from the most distal marker closest to the site of impact (i.e., the thenar region of the palm), which was then subsequently used for all remaining markers.

\section{Statistical Analysis}

Statistical analyses were executed using SPSS 24.0 (IBM SPSS Statistics, IBM Corporation, Somers, NY, USA). Independent samples t-tests were used to compare differences

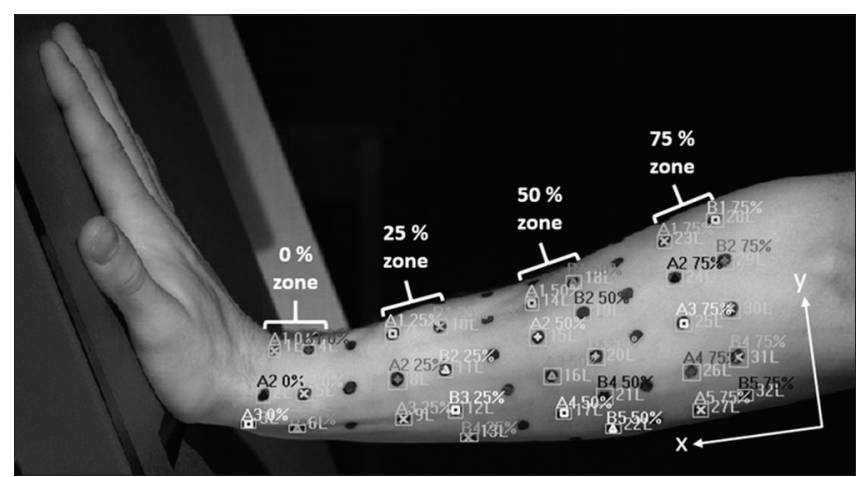

Figure 4. Screenshot from ProAnalyst ${ }^{\mathbb{B}}$ (zoomed in) showing the two columns of markers (A and B) selected for the $0 \%, 25 \%, 50 \%$, and $75 \%$ zones.

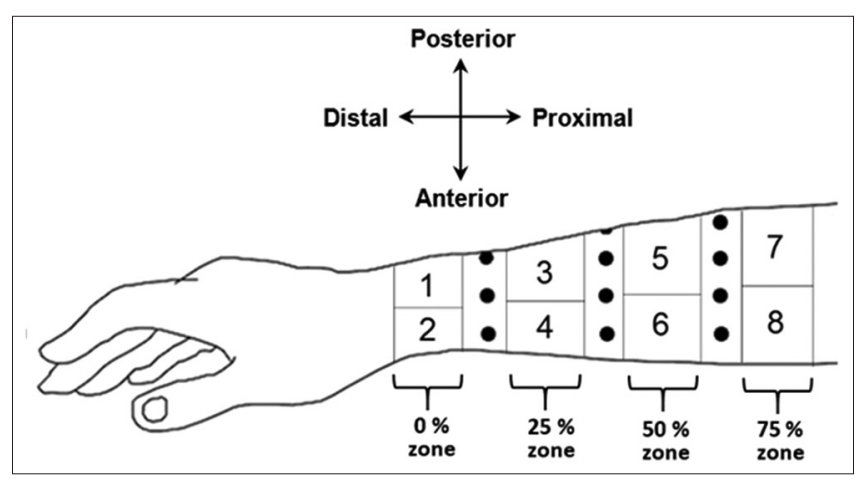

Figure 5. Schematic diagram of the marker grid $(2 \times 2 \mathrm{~cm}$ squares of dots) and the eight regions within the four analysis zones $(0 \%, 25 \%, 50 \%$, and $75 \%)$ on the forearm. 
in age, height, and body mass between skin pigmentation groups. Mean peak values for each impact response parameter (displacement, velocity, rebound distance) were obtained by averaging participants' peak values across the three impact trials from the SLU marker design in the proximal-distal and anterior-posterior directions for each region. Two-way mixed ANOVAs (between-subject factor: sex (female, male); within-subject factor: forearm region (1-8)) were used to examine if the impact responses (from the SLU impact trials) differed between sexes depending on the region of the forearm being tracked. Two-way mixed ANOVAs (between-subject factor: skin pigmentation (light, dark); within-subject factor: marker design (SLU, SNU)) were also performed to examine if the impact responses differed between the light and dark skin pigmentation groups depending on the marker design applied to the skin; data were collapsed across the eight forearm regions and the mean peak soft tissue impact responses of the three impact trials for each marker design (SLU and SNU) were used.

A value of alpha as 0.05 was implemented for all statistical comparisons. Normality of all soft tissue impact responses was assessed using Shapiro-Wilk tests and Q-Q Plots. Variance assumptions for between- and within-subject factors were assessed using Levene's Test for Equality of Variance and Mauchly's Test of Sphericity, respectively. Bonferroni Post Hoc tests for pairwise comparisons were performed for any significant main effects that were found, and if any significant interactions were revealed, simple effects tests were conducted.

\section{RESULTS}

Preliminary screening of the forearm impact responses revealed that two male participants in the light pigmentation group had extreme outliers $(z$-score $>3.29)$ with respect to anterior soft tissue displacement. Visual inspection of the automated motion tracking recordings in ProAnalyst ${ }^{\circledR}$ veri- fied that these values were not representative of genuine soft tissue motion, but rather, downward movement of the entire forearm due to moderate elbow flexion after impact. Since this violated the impact protocol guidelines, their data were excluded from all subsequent analyses.

No significant differences were found between the two skin pigmentation groups in terms of age, height, and body mass across all participants, and when split by sex $(p>0.05)$. All impact responses were approximately normally distributed, as assessed by Shapiro-Wilk tests $(p>0.05)$ and QQ Plots, with the exception of mean peak proximal and posterior displacement. Automated tracking of the soft tissue motion showed that the majority of markers did not return past the onset point in these two directions (see Figure 6), producing frequent zero values. This resulted in very positively skewed distributions with mean peak displacements

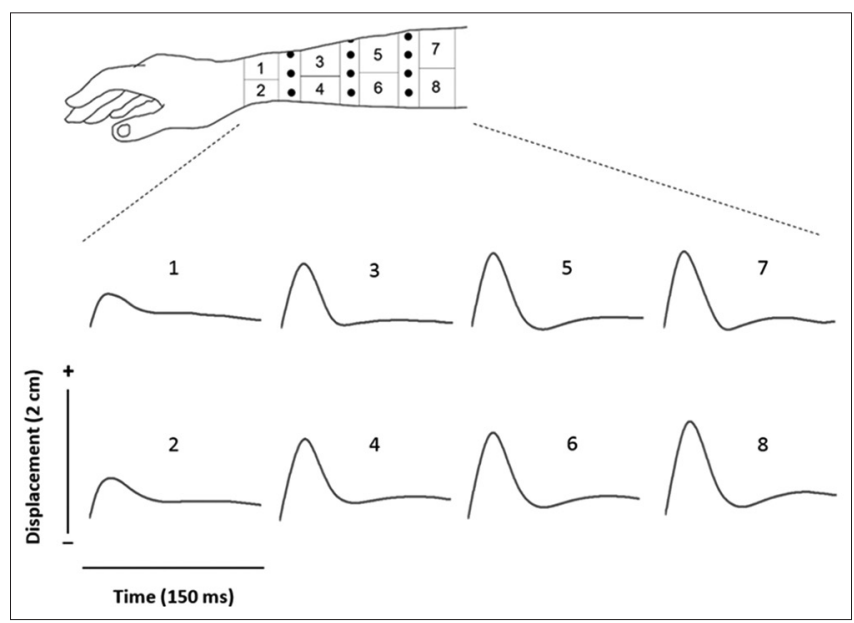

Figure 6. Sample displacement/time responses (proximal-distal axis) for each of the eight regions across the forearm for a single trial from one participant. The responses from each region have been aligned in time and displacement in order to show the relative differences.

Table 1. Mean $( \pm \mathrm{SD})$ overall female and male peak soft tissue displacement $(\mathrm{cm})$ in the proximal, distal, anterior, and posterior directions for each of the four zones $(0 \%, 25 \%, 50 \%, 75 \%)$ and eight regions.

\begin{tabular}{|c|c|c|c|c|c|c|c|c|}
\hline \multirow[t]{3}{*}{ Direction } & \multicolumn{8}{|c|}{ Regions } \\
\hline & \multicolumn{2}{|c|}{$(0 \%)$} & \multicolumn{2}{|c|}{$(25 \%)$} & \multicolumn{2}{|c|}{$(50 \%)$} & \multicolumn{2}{|c|}{$(75 \%)$} \\
\hline & 1 & 2 & 3 & 4 & 5 & 6 & 7 & 8 \\
\hline \multicolumn{9}{|l|}{ Female } \\
\hline Distal & $0.67(0.12)$ & $0.78(0.15)$ & $1.14(0.15)$ & $1.29(0.17)$ & $1.16(0.17)$ & $1.37(0.18)$ & $1.12(0.18)$ & $1.52(0.18)$ \\
\hline Proximal & $0.03(0.05)$ & $0.01(0.03)$ & $0.11(0.15)$ & $0.03(0.09)$ & $0.13(0.15)$ & $0.07(0.11)$ & $0.07(0.12)$ & $0.05(0.13)$ \\
\hline Anterior & $0.18(0.08)$ & $0.18(0.08)$ & $0.52(0.16)$ & $0.47(0.19)$ & $0.91(0.28)$ & $0.77(0.35)$ & $1.29(0.46)$ & $1.07(0.51)$ \\
\hline Posterior & $0.09(0.09)$ & $0.06(0.10)$ & $0.05(0.09)$ & $0.06(0.10)$ & $0.06(0.12)$ & $0.06(0.13)$ & $0.07(0.14)$ & $0.08(0.15)$ \\
\hline \multicolumn{9}{|l|}{ Male } \\
\hline Distal & $0.61(0.13)$ & $0.71(0.11)$ & $1.05(0.25)$ & $1.10(0.24)$ & $1.07(0.26)$ & $1.23(0.28)$ & $1.06(0.23)$ & $1.39(0.30)$ \\
\hline Proximal & $0.07(0.11)$ & $0.04(0.09)$ & $0.05(0.09)$ & $0.03(0.07)$ & $0.05(0.10)$ & $0.02(0.06)$ & $0.03(0.08)$ & $0.01(0.03)$ \\
\hline Anterior & $0.33(0.13)$ & $0.30(0.13)$ & $0.60(0.14)$ & $0.53(0.19)$ & $1.00(0.38)$ & $0.90(0.40)$ & $1.36(0.45)$ & $1.13(0.53)$ \\
\hline Posterior & $0.07(0.08)$ & $0.07(0.09)$ & $0.05(0.09)$ & $0.06(0.09)$ & $0.04(0.08)$ & $0.05(0.08)$ & $0.04(0.08)$ & $0.06(0.11)$ \\
\hline
\end{tabular}

Distal=soft tissue motion toward the wrist; Proximal=soft tissue motion toward the elbow; Anterior=downward soft tissue motion perpendicular to the long axis of the forearm; Posterior=upward soft tissue motion perpendicular to the long axis of the forearm 
of $\leq 0.1 \mathrm{~cm}$ (Table 1$)$, which provided little information on the actual soft tissue motion occurring in the proximal and posterior directions. Consequently, proximal and posterior rebound distances were also analyzed. Variances of the soft tissue impact responses between females and males for all regions of the forearm were homogeneous $(p>0.05)$, apart from proximal displacement in regions $1(p=0.08)$ and 2 $(p=0.07)$, anterior displacement in region $1(p=0.023)$, and posterior rebound distance in regions $6(p=0.027)$ and 8 $(p=0.033)$. Within these regions, the variances for the males were approximately double that of the females, consistently. Mauchly's Test of Sphericity was found to be significant $(p<0.05)$ for each soft tissue impact response, therefore, corrected Greenhouse-Geisser estimates were used for all the following analyses.

\section{Displacement}

No significant sex differences in mean peak displacement were found in any of the directions (proximal, distal, anterior, posterior) analyzed when compared across the eight regions of the forearm $(p>0.05)$ (Table 1). With respect to the proximal-distal axis, the greatest peak displacement occurred in region 8 in the distal direction toward the wrist $(1.47 \mathrm{~cm})$; the greatest peak displacement along the anterior-posterior axis was in region 7 in the anterior direction $(1.32 \mathrm{~cm})$. In these two directions, a general trend of increasing displacements was observed moving from distal to proximal regions of the forearm (i.e., $0 \%$ to $75 \%$ zone) (Figure $7 \mathrm{a}$ and c). In contrast, peak displacements in both the proximal and posterior directions had very small magnitudes $(\leq 0.10 \mathrm{~cm}$ and $\leq 0.08 \mathrm{~cm}$, respectively) across all regions (Figure $7 \mathrm{~b}$ and $\mathrm{d}$ ).

Based on the forearm region measured, significant differences in peak displacement were found. For peak distal displacement, significant consecutive increases in displacement were seen across all anterior regions of the forearm $(2,4,6$, and 8$)(p<0.05)$, whereas posterior regions only showed one significant increase from region 1 to regions 3,5 , and 7 ( $p<0.05)$ (Figure 7a). Peak distal displacements demonstrated the greatest increase from the $0 \%$ zone $(0.70 \mathrm{~cm})$ to the $25 \%$ zone $(1.16 \mathrm{~cm})$ of approximately $66 \%$. On average, peak distal displacement in the anterior regions of the forearm was $19 \%$ greater than the posterior regions. Peak anterior displacement in anterior and posterior regions of the forearm, also showed significant increases, moving distally to proximally $(p<0.05$ ) (Figure $7 \mathrm{c}$ ). No significant differences were observed between anterior and posterior regions for multiple pairs (regions 1 and 2, regions 3 and 4, regions 5 and 8$)(p>0.05)$. Posterior forearm regions had $16 \%$ greater peak anterior displacement than anterior regions, on average, however.

\section{Rebound Distance}

A significant interaction was present between sex and forearm region for proximal rebound distance $[F(3.111,87.115)$ $=6.724, p<0.001$, partial $\left.\eta^{2}=0.194\right]$, in which females demonstrated significantly more soft tissue rebound proximally toward the elbow for regions 3 through 8 compared to males $(p<0.05)$ (Figure 8 a). Simple main effects showed that both sexes demonstrated similar patterns across forearm region: regions 1 and 2 had significantly lower magnitudes than the remaining regions (3-8), resulting in an approximate increase in proximal rebound distance of $81 \%$ and $56 \%$ for females and males, respectively, from the $0 \%$ to $25 \%$ zone. No interactions were found between sex and forearm region for peak posterior rebound distance, although the magnitudes of regions 1 and 2 were also significantly lower than all other regions of the forearm $(p<0.05)$ (Figure 8b).
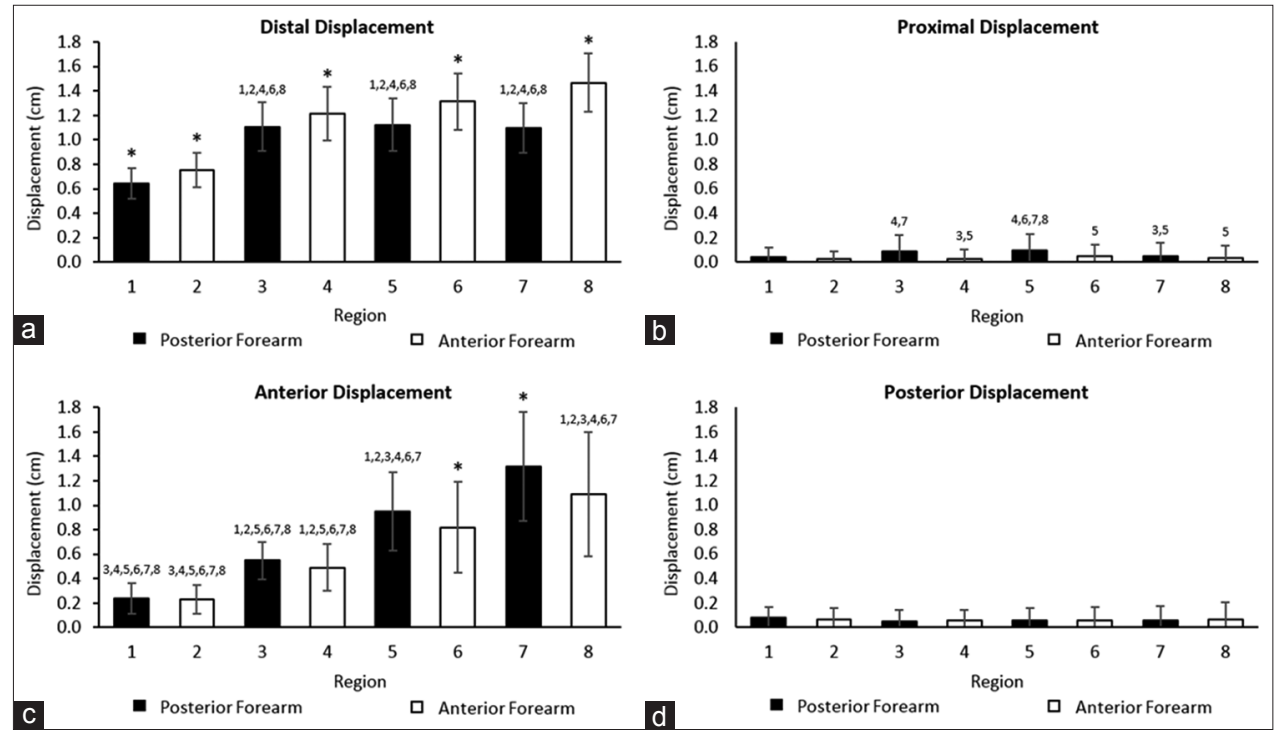

Figure 7. Mean (SD) peak soft tissue displacement (cm) in the (a) distal, (b) proximal, (c) anterior, and (d) posterior direction for all forearm regions $(1-8)$. Note. ${ }^{*}=$ significant difference from all regions $(p<0.05)$; each number represents a significant difference from that specific region $(p<0.05)$. 


\section{Velocity}

Mean peak velocities paralleled the displacement results (Table 2): no significant sex differences were found across the eight forearm regions in any of the directions analyzed $(p>0.05)$, and a general pattern of increasing velocities was observed when moving from distal to proximal regions (Figure 9). The greatest peak velocities occurred along the proximal-distal axis in the distal direction $(\geq 90.9 \mathrm{~cm} / \mathrm{s})$, with region 7 having the highest magnitude of $112.8 \mathrm{~cm} / \mathrm{s}$. For the anterior-posterior axis, region 7 also possessed the greatest peak velocity in the anterior direction, although, the magnitude was approximately half that of the highest distal velocity $(61.2 \mathrm{~cm} / \mathrm{s})$. The smallest peak velocities collectively occurred in the posterior direction $(\leq 27.6 \mathrm{~cm} / \mathrm{s})$.

With respect to forearm region, peak distal velocities in region 1 and region 7 had significantly lower and higher magnitudes than all other regions, respectively $(p<0.05)$, while the remaining regions demonstrated no significant differences across multiple pairs $(p>0.05)$ (Figure 9a). The effect of region on peak proximal velocity was comparable to proximal rebound distance, as proximal velocities in the $0 \%$ zone were also significantly lower than regions 3 through $8(p<0.05)$ (Figure 9b), resulting in an approximate increase of $85 \%$ when transitioning to the $25 \%$ zone. A significant interaction

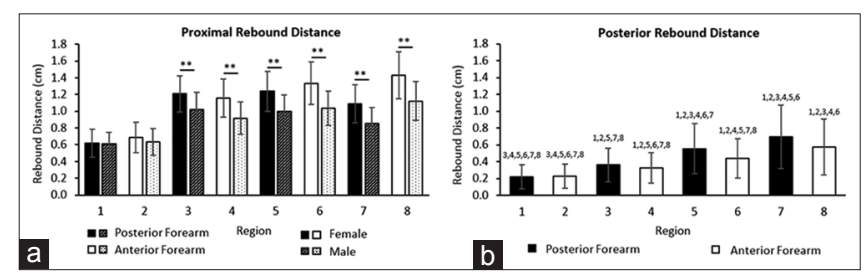

Figure 8. Mean (SD) peak soft tissue rebound distance $(\mathrm{cm})$ in the (a) proximal and (b) posterior direction for all forearm regions $(1-8)$. Note. $* *=$ significant difference between females and males $(P<0.05)$; each number represents a significant difference from that specific region $(P<0.05)$. between sex and forearm region $[F(2.556,71.566)=3.023$, $p=0.043$, partial $\left.\eta^{2}=0.097\right]$ for peak anterior velocity revealed significantly different regional comparisons across the forearm for females and males $(p<0.05)$, but no significant differences between the sexes for any regions $(p>0.05)$. Similar to anterior displacement, the peak anerior velocities in the posterior regions were increasingly higher than the anterior regions within the same zone moving proximally along the forearm (e.g., $25 \%$ zone: $+3 \%$; 50\% zone: $+16 \%$; $75 \%$ zone: $+24 \%$ ) (Figure 9c). For peak posterior velocity, no significant differences were observed in the distal half of the forearm (regions 1-4), however, in the proximal half of the forearm (regions 5-8), region $7(27.6 \mathrm{~cm} / \mathrm{s})$ had the significantly greatest posterior velocity compared to all other regions, except region $5(24.2 \mathrm{~cm} / \mathrm{s})$ (Figure $9 \mathrm{~d})$.

\section{Massless Skin Marker Designs}

Variances of all soft tissue impact responses between light and dark skin pigmentation groups for each marker design were homogeneous $(p>0.05)$. A significant interaction was found between skin pigmentation and marker design on peak distal displacement $[F(1.000,28.000)=4.530, p=0.042$, partial $\left.\eta^{2}=0.139\right]$. For the dark skin pigmentation group, distal displacement for the SLU marker design was $0.05 \mathrm{~cm}$ greater than the SNU marker design $(p<0.05)$; the light skin pigmentation group showed no significant differences $(p>0.05)$. A cross-over interaction for peak proximal displacement $[F(1.000,28.000)=5.554, p=0.026$, partial $\left.\eta^{2}=0.166\right]$ showed that the SLU marker design resulted in higher proximal displacement for the light skin pigmentation group and lower for the dark skin pigmentation group, compared to the SNU marker design, though, these differences were not significant $(p>0.05)$. A significant main effect of marker design on peak proximal rebound distance revealed that the SLU marker design $(1.02 \mathrm{~cm})$ had significantly greater soft tissue rebound in relation to the SNU marker design $(0.98 \mathrm{~cm})(p<0.05)$. Peak velocities in the distal,

Table 2. Mean $( \pm \mathrm{SD})$ overall female and male peak soft tissue velocity $(\mathrm{cm} / \mathrm{s})$ in the proximal, distal, anterior, and posterior directions for each of the four zones $(0 \%, 25 \%, 50 \%, 75 \%)$ and eight regions.

\begin{tabular}{|c|c|c|c|c|c|c|c|c|}
\hline \multirow[t]{3}{*}{ Direction } & \multicolumn{8}{|c|}{ Regions } \\
\hline & \multicolumn{2}{|c|}{$(0 \%)$} & \multicolumn{2}{|c|}{$(25 \%)$} & \multicolumn{2}{|c|}{$(50 \%)$} & \multicolumn{2}{|c|}{$(75 \%)$} \\
\hline & 1 & 2 & 3 & 4 & 5 & 6 & 7 & 8 \\
\hline \multicolumn{9}{|l|}{ Female } \\
\hline Distal & $88.9(13.4)$ & $96.6(12.8)$ & $100.6(12.4)$ & $103.4(12.6)$ & $105.5(14.4)$ & $104.3(13.1)$ & $110.3(14.7)$ & $107.6(13.4)$ \\
\hline Proximal & $32.7(10.3)$ & $29.7(9.7)$ & $61.2(11.5)$ & $55.3(6.9)$ & $57.0(9.8)$ & $57.5(7.8)$ & $45.9(11.0)$ & $59.8(10.9)$ \\
\hline Anterior & $32.1(8.5)$ & $34.3(9.5)$ & $42.8(10.4)$ & $43.2(10.7)$ & $58.9(12.9)$ & $48.3(13.0)$ & $63.8(15.7)$ & $49.3(15.5)$ \\
\hline Posterior & $20.3(8.7)$ & $15.6(7.0)$ & $15.1(6.1)$ & $16.8(7.7)$ & $25.9(10.4)$ & $18.0(7.3)$ & $30.4(11.3)$ & $19.8(8.8)$ \\
\hline \multicolumn{9}{|l|}{ Male } \\
\hline Distal & $94.0(14.0)$ & $103.6(12.9)$ & $108.7(15.2)$ & $110.3(14.0)$ & $112.9(17.4)$ & 111.7 (14.9) & $116.6(18.4)$ & $113.4(15.0)$ \\
\hline Proximal & $35.4(10.2)$ & $33.4(12.5)$ & $68.9(16.6)$ & $56.0(12.5)$ & $62.2(16.6)$ & $61.4(12.7)$ & $52.0(15.9)$ & $62.9(13.8)$ \\
\hline Anterior & $38.4(11.3)$ & $38.8(11.5)$ & $44.9(8.8)$ & $41.7(9.7)$ & $52.9(13.5)$ & $49.1(12.3)$ & $57.3(13.2)$ & $49.3(10.9)$ \\
\hline Posterior & $11.8(5.5)$ & $11.3(6.4)$ & $16.6(9.6)$ & $13.9(8.0)$ & $21.5(11.9)$ & $19.1(10.1)$ & $23.4(12.1)$ & $17.8(11.5)$ \\
\hline
\end{tabular}

Distal=soft tissue motion toward the wrist; Proximal=soft tissue motion toward the elbow; Anterior=downward soft tissue motion perpendicular to the long axis of the forearm; Posterior=upward soft tissue motion perpendicular to the long axis of the forearm 

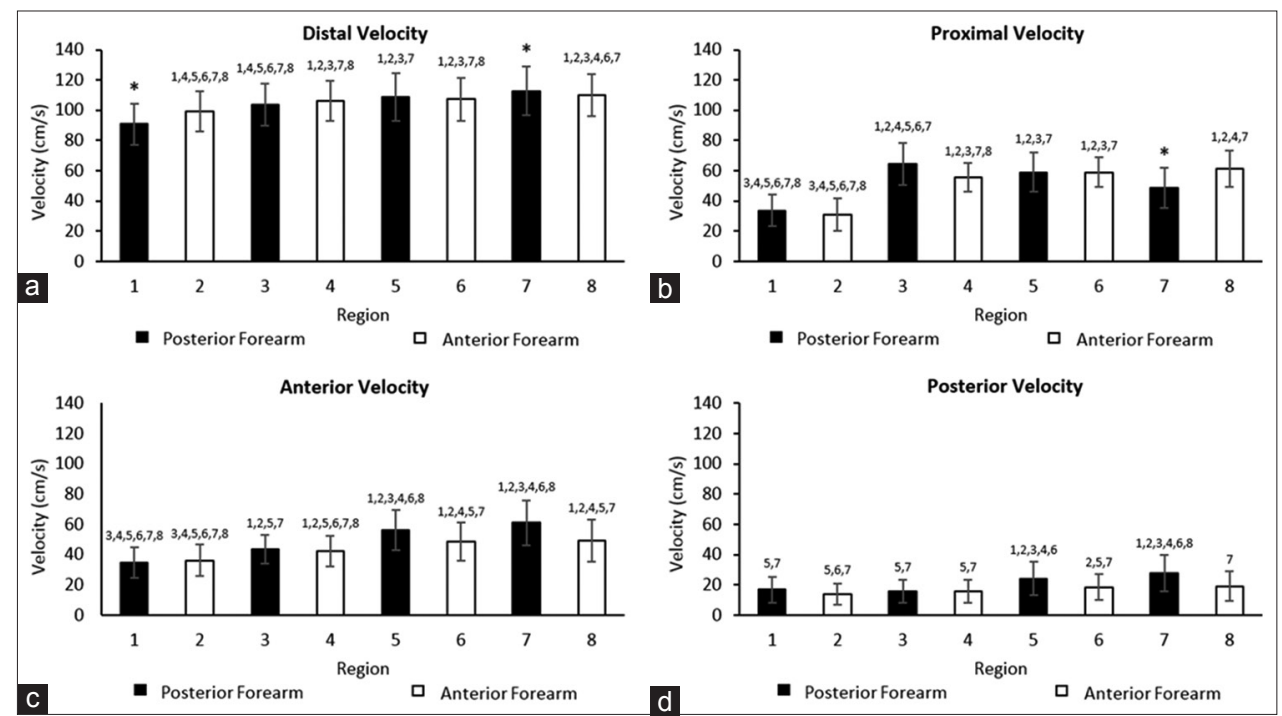

Figure 9. Mean (SD) peak soft tissue velocity ( $\mathrm{cm} / \mathrm{s}$ ) in the (a) distal, (b) proximal, (c) anterior, and (d) posterior direction for all forearm regions $(1-8)$. Note. $*=$ significant difference from all regions $(p<0.05)$; each number represents a significant difference from that specific region $(p<0.05)$.

proximal, and anterior directions also demonstrated similar trends in which the SLU marker design produced significantly higher velocities than the SNU marker design of $2.5 \mathrm{~cm} / \mathrm{s}$, $1.5 \mathrm{~cm} / \mathrm{s}$, and $1.5 \mathrm{~cm} / \mathrm{s}$, respectively $(p<0.05)$.

During automated motion tracking in ProAnalyst ${ }^{\circledR}$, one case occurred in which the defined region surrounding a SLU marker jumped to an adjacent marker mid-way through the automated tracking process; no tracking discrepancies occurred with the SNU markers. SLU markers located near the anterior and posterior edges of the forearm were found to be slightly more susceptible to marker drop out compared to the SNU markers, especially where minor shadowing underneath the forearm reduced the contrast on the anterior edge. Additionally, higher rates of marker drop out during automated tracking were observed for SLU markers located distally and posteriorly in the $0 \%$ zone of the forearm, as the smaller sized SLU markers $(0.5 \mathrm{~cm}$ diameter $)$ were more easily lost where the skin compressed near the wrist joint due to hyperextension; the larger sized SNU markers $(\sim 1 \mathrm{~cm}$ diameter) proved to be slightly more resilient to this issue.

\section{DISCUSSION}

In the present study, forearm soft tissue motion following forward fall hand impacts was able to be recorded and analyzed successfully using massless skin markers and automated motion tracking software. On average, the greatest peak soft tissue displacements and velocities occurred distally toward the wrist compared to all other directions, consistent with the findings by Stefanczyk et al. (2013) for leg soft tissue motion following heel impacts. The greatest distal displacement along the long axis of the forearm $(1.47 \mathrm{~cm})$ was slightly less than the maximum marker motion $(1.7 \mathrm{~cm})$ previously determined by Pain and Challis (2002), though, this is most likely attributable to the different impact scenarios utilized (e.g., simulated forward fall and active downward palm striking task, respectively). While no comparable velocity data currently exists for the upper extremity, the greatest distal velocity of leg soft tissue reported by Brydges et al. (2015) was found to be moderately lower or higher than that of the forearm $(112.8 \mathrm{~cm} / \mathrm{s})$ depending on the impact technique: horizontal pendulum impacts $(95.2 \mathrm{~cm} / \mathrm{s})$ and vertical drop tests $(137.5 \mathrm{~cm} / \mathrm{s})$.

Peak proximal (and posterior) displacements observed in this study were very limited given that the motion pathways of the selected markers (and thus the underlying forearm soft tissues) often did not return past the onset point (Figure 6). This is in contrast to the underdamped oscillatory response seen by Pain and Challis (2002). Despite providing both a practical and reputable method for simulating real-life forward fall events that has been frequently used in past literature (Kim \& Ashton-Miller, 2003; Hwang et al., 2006; Lattimer et al., 2016; Lattimer et al., 2017), the design of the torso-release apparatus, in combination with the effects of gravity, may have contributed to these results since the final orientation of the forearm at impact was relatively horizontal. This would likely pull the soft tissues towards the ground (i.e., anteriorly), limiting soft tissue recovery in the proximal and posterior directions.

Despite clear differences in upper extremity soft and rigid tissue masses between females and males (Maughan, Abel, Watson \& Weir, 1986; Mazess, Barden, Bisek \& Hanson, 1990), the general lack of sex differences across the impact responses in this study suggests that forearm soft tissue motion associated with forward fall hand impacts may not be driven by tissue composition, but rather, the distribution of soft tissues along the forearm. Only proximal rebound distance showed significant differences between sexes, where females had approximately $25 \%$ greater proximal rebound in intermediate and distal forearm regions (3-8) compared to males. However, due to insufficient space for marker placement on the hand, palm deformation could not be measured in the current study as Brydges et al. (2015) did for the heel pad, to verify its contribution to the impact responses between females and males. To date, palm soft tissue thickness has only been quantified by ultrasound for young women (Choi \& Robinovitch, 2011). Therefore, assuming that the 
palmar soft tissues for males are thicker than for females, similar to what has been shown for the heel pad of the foot (Prichasuk, 1994), this would help explain the reduced proximal motion of the forearm soft tissues for males, as more impact shock would be absorbed at the hand as the palm compresses.

The increase in regional impact responses observed moving distally to proximally further supports the notion that the movement of the forearm soft tissues after a forward fall hand impact is, to some degree, a function of tissue distribution along the segment, especially with the greatest impact responses commonly occurring in regions 7 and 8 where the most tissue is located. In addition, notably sharp increases from the $0 \%$ to $25 \%$ zones for distal displacement, proximal rebound distance, and proximal velocity highlight the potential importance of this section of the distal forearm when analyzing shock attenuation in the body related to forward falls. Given the high incidence of distal radius fractures occurring at or near this location (Nellans, Kowalski \& Chung, 2012), more research is needed to better understand the protective mechanisms of the underlying tissues occurring here.

Overall, the magnitudes of the statistical differences between the soft tissue impact responses for the SLU and SNU marker designs were extremely small (displacements: $\leq 0.05 \mathrm{~cm}$; rebound distances: $\leq 0.04 \mathrm{~cm}$; velocities: $\leq$ $2.5 \mathrm{~cm} / \mathrm{s}$ ). As a result, for the purpose of automatically tracking soft tissue motion across varying skin pigmentations, it is suggested that the overall precision of each marker design is functionally equivalent. The greater shape variation of the SNU markers did not have as beneficial of an effect as what has been previously reported for other non-contact motion tracking methods, such as digital image correlation (Haddadi \& Belhabib, 2008; Crammond et al., 2013), and enhancements to marker contrast offered only minimal improvements to the automatic tracking process (i.e., marker drop out) when minor shadowing and marker placement were taken into account. Therefore, based on the practicality of each approach, the SLU marker design may prove to be superior since it was more time- and cost-effective than the SNU marker design (i.e., applying only one layer compared to two; using a standard permanent marker pen compared to more expensive, specialty water-based paint marker pens). However, the self-report format of the Fitzpatrick Skin Type Questionnaire relies heavily on a subjective evaluation of oneself (e.g., does your skin tan?), so it is possible that some participants may have been falsely categorized (specifically in the dark skin pigmentation group) due to over-estimating their skin's reactivity to the sun, reducing the need for markers with enhanced contrast. Consequently, the validity of this questionnaire for categorizing skin pigmentations should be addressed if it is to be used to determine contrast requirements for soft tissue motion capture, as done in this study.

A vital assumption of using massless skin markers to quantify soft tissue movement following impact is that the superficial soft tissues (i.e., skin) move synchronously with the underlying deep soft tissues (i.e., muscle and fat), and thus, produce identical impact responses relative to bone (Brydges et al., 2015). Since this could not be tested in the current study, given the superficial and non-invasive nature of the approaches used, it could be viewed as a potential source of measurement error. Furthermore, the motion of the rigid tissue (i.e., bone) was not directly measured. As a result, the intra-segmental marker motion may have been influenced, to a certain extent, by the whole limb motion of the forearm (Pain \& Challis, 2002). However, the impact of this limitation was minimised by carefully instructing participants to maintain proper upper extremity posture during data collection and inspecting the captured video footage after each impact trial. In addition, factors that have been shown to influence impact shock attenuation in the upper extremity, such as muscle activation levels (Pain \& Challis, 2002; Burkhart \& Andrews, 2010a) and joint angles (DeGoede, Ashton-Miller, Schultz \& Alexander, 2002b), were not directly controlled for, as quantifying these measures without the use of devices which need to be externally affixed to the skin (e.g., electromyography, electrogoniometers, etc.) was a challenge that could not be met during the current study.

The generalizability of the results of this study is limited to a younger adult population (17-30 years of age). It is likely that the significant changes in body composition, such as sarcopenia (Baumgartner, 2000) and progressive declines of skin elasticity (Sumino et al., 2004; Luebberding, Krueger \& Kerscher, 2014) that occur with age, would influence the forearm soft tissue impact responses of people older than the participants in this study. This will have implications for modelling segmental responses following impact events across different age groups. Considering that current wobbling mass biomechanical models include very simplified soft tissue components with respect to their shape and motion characteristics (Gruber, Ruder, Denoth \& Schneider, 1998; Gittoes et al., 2006; Pain and Challis, 2006), further examination of the relative motions of soft tissue elements within individual segments of different aged participants will help improve the biofidelity and generalizability of future biomechanical modeling efforts.

\section{CONCLUSIONS}

In summary, as suggested by Pain and Challis (2002), ignoring the importance of soft tissue motion for attenuating impact shock in the body, and dismissing it as error (i.e., soft tissue artifact) that needs to be removed from biomechanical analyses (Peters, Galna, Sangeux, Morris \& Baker, 2010), may limit our knowledge of the injury mechanisms at play during dynamic impact events. To the authors' knowledge, this is the first study to quantify the motion associated with the impact response of the forearm soft tissues following a forward fall onto the hands of outstretched arms. Consequently, this research provides novel insight into the shock attenuating characteristics of the soft tissues in the forearm, wherein peak soft tissue displacement and velocity were found to generally increase moving distally to proximally along the forearm, with the greatest impact responses occurring in the distal direction; females and males showed no significant differences for this body segment, on average. Future work should look to better comprehend how soft tissue responds both independently and in conjunction with other injury 
prevention strategies proven to help mitigate impact forces, such as wrist guards (Burkhart \& Andrews 2010b) and energy-absorbing flooring systems (Laing \& Robinovitch, 2009). Moreover, with multiple studies investigating both in- and out-of-plane motion of soft tissues (Manal, McClay Davis, Galinat \& Stanhope, 2003; Stagni, Fantozzi, Cappello \& Leardini, 2005; Akbarshahi et al., 2010; Mills, Scurr \& Wood, 2011), 3D analysis utilizing either of the marker setups evaluated in the current study, would be a logical next step to advance the scope of this research, and to fully understand the effect that soft tissue motion has on the propagation and attenuation of impact forces in the forearm and body.

\section{ACKNOWLEDGEMENTS}

The financial support from NSERC is gratefully acknowledged as well as the efforts of Lauren Gyemi and Mary Birkner for their assistance with pilot data collection and data analysis.

\section{REFERENCES}

Akbarshahi, M., Schache, A., Fernandez, J., Baker, R., Banks, S., \& Pandy, M. (2010). Non-invasive assessment of soft-tissue artifact and its effect on knee joint kinematics during functional activity. Journal of Biomechanics, 43(7), 1292-1301.

Baumgartner, R. N. (2000). Body composition in healthy aging. Annals of the New York Academy of Sciences, 904, 437-448.

Brydges, E. A., Burkhart, T. A., Altenhof, W. J., \& Andrews, D. M. (2015). Leg soft tissue position and velocity data from skin markers can be obtained with good to acceptable reliability following heel impacts. Journal of Sports Sciences, 33(15), 1606-1613.

Burkhart, T. A., \& Andrews, D. M. (2010a). Activation level of extensor carpi ulnaris affects wrist and elbow acceleration responses following simulated forward falls. Journal of Electromyography and Kinesiology, 20(6), 1203-1210.

Burkhart, T. A., \& Andrews, D. M. (2010b). The effectiveness of wrist guards for reducing wrist and elbow accelerations resulting from simulated forward falls. Journal of Applied Biomechanics, 26(3), 281-292.

Burkhart, T. A., Andrews, D. M., \& Dunning, C. E. (2012). Failure characteristics of the isolated distal radius in in response to dynamic impact loading. Journal Orthopaedic Research, 30(6), 885-892.

Burkhart, T. A., Quenneville, C. E., Dunning, C. E., \& Andrews, D. M. (2014). Development and validation of a distal radius finite element model to simulate impact loading indicative of a forward fall. Proceedings of the Institution of Mechanical Engineers. Part H, Journal of engineering in medicine, 228(3), 258-271.

Choi W. J., \& Robinovitch, S. N. (2011). Pressure distribution over the palm region during forward falls on the outstretched hands. Journal of Biomechanics, 44(3), 532-539.

Cole, G. K., Nigg, B. M., van den Bogert, A. J., \& Gerritsen, K. G. M. (1996). Lower extremity joint load- ing during impact in running. Clinical Biomechanics, 11(4), 181-193.

Crammond, G., Boyd, S. W., \& Dulieu-Barton, J. M. (2013). Speckle pattern quality assessment for digital image correlation. Optics and Lasers in Engineering, 51(12), 1368-1378.

DeGoede, K. M., \& Ashton-Miller, J. A. (2002a). Fall arrest strategy affects peak hand impact force in a forward fall. Journal of Biomechanics, 35(6), 843-848.

DeGoede, K. M., Ashton-Miller, J. A., Schultz, A. B., \& Alexander, N. B. (2002b). Biomechanical factors affecting the peak hand reaction force during the bimanual arrest of a moving mass. Journal of Biomechanical Engineering, 124(1), 107-112.

Fitzpatrick, T. B. (1988). The validity and practicality of sun reactive skin types I through VI. Archives of Dermatology, 124(6), 869-871.

Fuller, J., Liu, L., Murphy, M., \& Mann, R. (1997). A comparison of lower-extremity skeletal kinematics measured using skin- and pin-mounted markers. Human Movement Science, 16(2-3), 219-242.

Gao, B., \& Zheng, N. (2008). Investigation of soft tissue movement during level walking: translations and rotations of skin markers. Journal of Biomechanics, 41(15), 3189-3195.

Gittoes, M. J., Brewin, M. A., \& Kerwin, D. G. (2006). Soft tissue contributions to impact forces simulated using a four-segment wobbling mass model of forefoot-heel landings. Human Movement Science, 25(6), 775-787.

Greenwald, R. M., Janes, P. C., Swanson, S. C., \& McDonald, T. R. (1998). Dynamic impact response of human cadaveric forearms using a wrist brace. American Journal of Sports Medicine, 26(6), 825-830.

Gruber, K., Ruder, H., Denoth, J., \& Schneider, K. (1998). A comparative study of impact dynamics: wobbling mass model versus rigid body models. Journal of Biomechanics, 31(5), 439-444.

Haddadi, H., \& Belhabib, S. (2008). Use of rigid-body motion for the investigation and estimation of the measurement errors related to digital image correlation technique. $O p$ tics and Lasers in Engineering, 46(2), 185-196.

Hwang, I. K., Kim, K. J., Kaufman, K. R., Cooney, W. P., \& An, K. N. (2006). Biomechanical efficiency of wrist guards as a shock isolator. Journal of Biomechanical Engineering, 128(2), 229-234.

Idzikowski, J. R., Janes, P. C., \& Abbott, P. J. (2000). Upper extremity snowboarding injuries. Ten-year results from the Colorado snowboard injury survey. American Journal of Sports Medicine, 28(6), 825-832.

Kim, K. J., \& Ashton-Miller, J. A. (2003). Biomechanics of fall arrest using the upper extremity: Age differences. Clinical Biomechanics (Bristol, Avon), 18(4), 311-318.

Kuo, M. Y., Tsai, T. Y., Lin, C. C., Lu, T. W., Hsu, H. C., \& Shen, W. C. (2011). Influence of soft tissue artifacts on the calculated kinematics and kinetics of total knee replacements during sit-to-stand. Gait \& Posture, 33(3), 379-384

Laing, A. C., \& Robinovitch, S. N. (2009). Low stiffness floors can attenuate fall-related femoral impact forces 
by up to $50 \%$ without substantially impairing balance in older women. Accident; Analysis and Prevention, 41(3), 642-650.

Lattimer, L. J., Lanovaz, J. L., Farthing, J. P., Madill, S., Kim, S., \& Arnold, C. (2016). Upper limb and trunk muscle activation during an unexpected descent on the outstretched hands in young and older women. Journal of Electromyography and Kinesiology, 30, 231-237.

Lattimer, L. J., Lanovaz, J. L., Farthing, J. P., Madill, S., Kim, S., Robinovitch, S., \& Arnold, C. (2017). Female age-related differences in biomechanics and muscle activity during descents on the outstretched arms. Journal of Aging and Physical Activity, 25(3), 474-481.

Leardini, A., Chiari, L., Croce, U., \& Cappozzo, A. (2005). Human movement analysis using stereophotogrammetry: Part 3. Soft tissue artifact assessment and compensation. Gait \& Posture, 21(2), 212-225.

Luebberding, S., Krueger, N., \& Kerscher, M. (2014). Mechanical properties of human skin in vivo: a comparative evaluation in 300 men and women. Skin Research and Technology, 20(2), 127-135.

Manal, K. K., McClay Davis, I. I., Galinat, B. B., \& Stanhope, S. S. (2003). The accuracy of estimating proximal tibial translation during natural cadence walking: bone vs. skin mounted targets. Clinical Biomechanics (Bristol, Avon), 18(2), 126-131.

Maughan, R. J., Abel, R. W., Watson, J. S., \& Weir, J. (1986). Forearm composition and muscle function in trained and untrained limbs. Clinical Physiology, 6(4), 389-396.

Mazess, R. B., Barden, H. S., Bisek, J. P., \& Hanson, J. (1990). Dual-energy x-ray absorptiometry for total-body and regional bone-mineral and soft-tissue composition. American Journal of Clinical Nutrition, 51(6), 1106-1112.

Mills, C., Scurr, J., \& Wood, L. (2011). A protocol for monitoring soft tissue motion under compression garments during drop landings. Journal Biomechanics, 44(9), 1821-1823.

Mirhadi, S., Ashwood, N., \& Karagkevrekis, B. (2015). Review of rollerblading injuries. Trauma, 17(1), 29-32.

Muller, M. E., Webber, C. E., \& Bouxsein, M. L. (2003). Predicting the failure load of the distal radius. Osteoporosis International, 14(4), 345-352.

Myers, E., Sebeny, E., Hecker, A., Corcoran, T., Hipp, J., Greenspan, S., \& Hayes, W. (1991). Correlations between photon absorption properties and failure load of the distal radius in vitro. Calcified Tissue International, 49(4), 292-297.

Nellans, K. W., Kowalski, E., \& Chung, K. C. (2012). The epidemiology of distal radius fractures. Hand Clinics, 28(2), 113-125.

Nevitt, M. C., \& Cummings, S. R. (1993). Type of fall and risk of hip and wrist fractures: The study of osteoporotic fractures. The Study of Osteoporotic Fractures Research Group. Journal of the American Geriatrics Society, 41(11), 1226-1234

Pain, M. T., \& Challis, J. H. (2002). Soft tissue motion during impacts: Their potential contributions to energy dissipa- tion. Journal of Applied Biomechanics, 18(3), 231-242.

Pain, M. T., \& Challis, J. H. (2006). The influence of soft tissue movement on ground reaction forces, joint torques and joint reaction forces in drop landings. Journal of Biomechanics, 39(1), 119-124.

Palvanen, M., Kannus, P., Parkkari, J., Pitkäjärvi, T., Pasanen, M., Vuori, I., \& Järvinen, M. (2000). The injury mechanisms of osteoporotic upper extremity fractures among older adults: A controlled study of 287 consecutive patients and their 108 controls. Osteoporosis International, 11(10), 822-831.

Peters, A., Galna, B., Sangeux, M., Morris, M., \& Baker, R. (2010). Quantification of soft tissue artifact in lower limb human motion analysis: A systematic review. Gait \& Posture, 31(1), 1-8.

Prichasuk, S. (1994). The heel pad in plantar heel pain. Journal of Bone and Joint Surgery. British Volume, 76(1), 140-142.

Robinovitch, S. N., \& Chiu, J. (1998). Surface stiffness affects impact force during a fall on the outstretched hand. Journal of Orthopaedic Research, 16(3), 309-313.

Sangeux, M. M., Marin, F. F., Charleux, F. F., Dürselen, L. L., \& Ho Ba Tho, M. C. (2006). Quantification of the 3D relative movement of external marker sets vs. bones based on magnetic resonance imaging. Clinical Biomechanics (Bristol, Avon), 21(9), 984-991.

Sati, M., de Guise, J. A., Larouche, S., \& Drouin, G. (1996). Quantitative assessment of skin-bone movement at the knee. The Knee, 3(3), 121-138.

Stagni, R., Fantozzi, S., Cappello, A., \& Leardini, A. (2005). Quantification of soft tissue artefact in motion analysis by combining 3D fluoroscopy and stereophotogrammetry: A study on two subjects. Clinical Biomechanics (Bristol, Avon), 20(3), 320-329.

Stefanczyk, J. M., Brydges, E. A., Burkhart, T. A., Altenhof, W. J., \& Andrews, D. M. (2013). Surface accelerometer fixation method affects leg soft tissue motion following heel impacts. International Journal of Kinesiology and Sports Science, 1(3), 1-8.

Südhoff, I., Van Driessche, S., Laporte, S., de Guise, J.A., \& Skall, W. (2007). Comparing three attachment systems used to determine knee kinematics during gait. Gait \& Posture, 25(4), 533-543.

Sumino, H., Ichikawa, S., Abe, M., Endo, Y., Ishikawa, O., \& Kurabayashi, M. (2004). Effects of aging, menopause, and hormone replacement therapy on forearm skin elasticity in women. Journal of the American Geriatrics Society, 52(6), 945-949.

Winter, D. A. (2005). Biomechanics and motor control of human movement ( $2^{\text {nd }}$ ed). Hoboken, NJ: John Wiley and Sons Inc.

Wolf, A., \& Senesh, M. (2011). Estimating joint kinematics from skin motion observation: modelling and validation. Computer Methods in Biomechanics and Biomedical Engineering, 14(11), 939-946.

Wrbaškić, N. N., \& Dowling, J. J. (2007). An investigation into the deformable characteristics of the human foot using fluoroscopic imaging. Clinical Biomechanics (Bristol, Avon), 22(2), 230-238. 